\title{
The unique brain anatomy of meditation practitioners: alterations in cortical gyrification
}

\author{
Eileen Luders ${ }^{1}{ }^{*}$, Florian Kurth $^{2}$, Emeran A. Mayer ${ }^{2}$, Arthur W. Toga ${ }^{1}{ }^{*}$, Katherine L. Narr ${ }^{1}$ and \\ Christian Gaser ${ }^{3,4}$
}

1 Laboratory of Neuro Imaging, Department of Neurology, UCLA School of Medicine, Los Angeles, CA, USA

${ }^{2}$ Department of Medicine, Center for Neurobiology of Stress, UCLA School of Medicine, Los Angeles, CA, USA

${ }^{3}$ Department of Psychiatry, University of Jena, Jena, Germany

${ }^{4}$ Department of Neurology, University of Jena, Jena, Germany

\section{Edited by:}

Amishi P. Jha, University of Miami,

USA

\section{Reviewed by:}

Lutz Jäncke, University of Zurich, Switzerland

Donna R. Roberts, Medical University of South Carolina, USA

\section{*Correspondence:}

Eileen Luders and Arthur W. Toga, Laboratory of Neuro Imaging,

Department of Neurology, UCLA

School of Medicine, 635 Charles

Young Drive South, Suite 225, LoS

Angeles, CA 90095-7334, USA.

e-mail: eileen@loni.ucla.edu;

toga@loni.ucla.edu
Several cortical regions are reported to vary in meditation practitioners. However, prior analyses have focused primarily on examining gray matter or cortical thickness. Thus, additional effects with respect to other cortical features might have remained undetected. Gyrification (the pattern and degree of cortical folding) is an important cerebral characteristic related to the geometry of the brain's surface. Thus, exploring cortical gyrification in long-term meditators may provide additional clues with respect to the underlying anatomical correlates of meditation. This study examined cortical gyrification in a large sample ( $n=100)$ of meditators and controls, carefully matched for sex and age. Cortical gyrification was established by calculating mean curvature across thousands of vertices on individual cortical surface models. Pronounced group differences indicating larger gyrification in meditators were evident within the left precentral gyrus, right fusiform gyrus, right cuneus, as well as left and right anterior dorsal insula (the latter representing the global significance maximum). Positive correlations between gyrification and the number of meditation years were similarly pronounced in the right anterior dorsal insula. Although the exact functional implications of larger cortical gyrification remain to be established, these findings suggest the insula to be a key structure involved in aspects of meditation. For example, variations in insular complexity could affect the regulation of well-known distractions in the process of meditation, such as daydreaming, mind-wandering, and projections into past or future. Moreover, given that meditators are masters in introspection, awareness, and emotional control, increased insular gyrification may reflect an integration of autonomic, affective, and cognitive processes. Due to the cross-sectional nature of this study, further research is necessary to determine the relative contribution of nature and nurture to links between cortical gyrification and meditation.

Keywords: brain, cortical complexity, curvature, folding, insula, meditation, mindfulness, MRI

\section{INTRODUCTION}

Various brain regions have been reported to be anatomically different between meditators and controls, to show associations with the amount of meditation practice, and/or to change as a consequence of meditation exercises (Lazar et al., 2005; Pagnoni and Cekic, 2007; Holzel et al., 2008, 2010; Vestergaard-Poulsen et al., 2008; Luders et al., 2009, 2011a,b; Grant et al., 2010; Tang et al., 2010). Many of these aforementioned effects were evident across the lateral and medial surfaces of the cortex as well as buried beneath it (e.g., within the insula) suggesting the cerebral cortex to be heavily involved in processes related to meditation. Given that most prior analyses have focused on examining gray matter (GM) attributes (including cortical thickness), effects with respect to other cortical features might have remained undetected. Gyrification (the pattern and degree of cortical folding) is an important cerebral characteristic related to the geometry of the brain's surface. Thus, establishing profiles of cortical gyrification in meditators and comparing these profiles to well-matched controls may provide additional clues with respect to the underlying anatomical correlates of meditation. In addition, investigating associations between local gyrification and the amount of meditation experience may provide insights with respect to meditation-related neuronal plasticity (i.e., the brain's ability to change throughout life). Meditation practitioners who are seriously committed to a long-term practice provide an ideal human model for investigating brain plasticity given their ongoing, frequent, and regular mental efforts.

We applied a well-established and automated whole-brain approach to measure cortical gyrification at thousands of points across the cortical surface (Gaser et al., 2006; Luders et al., 2006, 2008). As described previously (Luders et al., 2006), our gyrification measure resembles the 3D extension of the wellknown gyrification index (GI; Zilles et al., 1988) defined as the ratio of the length of a $2 \mathrm{D}$ curve to the length of its convex hull. Several alternative approaches to analyze cortical folding/complexity have also been described (Awate et al., 2010). 
Traditionally, gyrification descriptors characterize cortical folding/complexity by a single number, either for the whole brain or for specific regions-of-interest (ROIs). While ROI-based measures provide a better regional specificity than whole-brain indices, measurements are still averaged across pre-defined regions. Moreover, anatomically defined boundaries do not always correspond to functionally relevant boundaries. In contrast, the current approach generates detailed and regionally specific estimates of surface complexity, at every point across the entire cortex, without requiring any a priori ROI definitions. Moreover, it is sensitive to the magnitude of folding (depths of sulci/heights of gyri) as well as to the frequency of folding (number of gyri/sulci).

The present study, which includes 100 subjects (i.e., 50 meditators and 50 controls, well-matched for gender and age), comprises the largest meditation sample investigated to date with structural imaging methods. Moreover, it constitutes the first study to address links between meditation and cortical gyrification. Though our hypotheses were two-tailed with respect to the direction of effects, we hypothesized that group differences and correlations would occur in brain regions implicated in prior meditation studies that have included other cortical measures (i.e., cortical thickness, GM volume, GM concentration/density). Specifically, we hypothesized that group differences and/or correlations would be most pronounced within the following cortical regions: (1) right (anterior) insula (Lazar et al., 2005; Holzel et al., 2008), (2) right (orbito-) frontal cortex (Lazar et al., 2005; Holzel et al., 2008; Luders et al., 2009), (3) left frontal cortex (Vestergaard-Poulsen et al., 2008), (4) left inferior temporal gyrus (Holzel et al., 2008; Luders et al., 2009), (5) left fusiform gyrus (Vestergaard-Poulsen et al., 2008), (6) bilateral paracentral lobe, including primary and secondary somato-sensory cortex (Luders et al., 2009; Grant et al., 2010) as well as (7) bilateral cingulate (Grant et al., 2010; Holzel et al., 2011).

\section{MATERIALS AND METHODS SUBJECTS}

Our study included 50 meditators and 50 control subjects which were matched pair-wise for sex (28 men, 22 women) and for age [meditators (mean $\pm \mathrm{SD}$ ): $51.4 \pm 12.8$ years; controls (mean \pm SD): $50.4 \pm 11.8$ years]. Age ranged between 24 and 71 years; the average age difference within a sex-matched pair was 1.8 years. While the scans for the controls were obtained from the ICBM database of normal adults ${ }^{1}$, meditators were newly recruited from various meditation venues. Years of meditation practice ranged between 4 and 46 years (mean \pm SD: $19.8 \pm 11.4$ years). An overview with respect to the subject-specific meditation style as well as amount of practice (i.e., number of years, frequency per week, and duration per session) is provided in Table A1 in the Appendix. The majority of subjects (89\%) were right-handed as based on self-reports of hand preference for selected activities. Six meditators and five controls were left-handed. All subjects were required to be free of any neurological and psychiatric disorders and gave informed consent according to UCLA's Institutional Review Board.

\footnotetext{
${ }^{1}$ http://www.loni.ucla.edu/ICBM/Databases/
}

\section{IMAGE ACQUISITION}

Magnetic resonance imaging (MRI) data from all subjects was acquired on a $1.5 \mathrm{~T}$ Siemens Sonata scanner (Erlangen, Germany) using an eight-channel head coil and a 3D T1weighted sequence (MPRAGE) with the following parameters: $\mathrm{TR}=1900 \mathrm{~ms}$, $\mathrm{TE}=4.38 \mathrm{~ms}$, flip angle $=15^{\circ}, 160$ contiguous $1 \mathrm{~mm}$ sagittal slices, FOV: $256 \mathrm{~mm} \times 256 \mathrm{~mm}$, matrix: $256 \times 256$, voxel dimensions: $1.0 \mathrm{~mm} \times 1.0 \mathrm{~mm} \times 1.0 \mathrm{~mm}$.

\section{IMAGE PROCESSING}

As detailed elsewhere (Dale et al., 1999; Fischl et al., 1999a,b), image volumes passed through a number of preprocessing steps using mostly automated procedures included in the FreeSurfer software suite, version $4.5^{2}$. Briefly, images were processed using intensity normalization and skull stripping, followed by linear registration and non-linear morphing to a probabilistic brain atlas, as well as tissue classification into GM, white matter (WM), CSF, and background. Subsequently, a WM surface was derived from the WM segment using the marching cubes algorithm, followed by topology correction. Then, by outwardly deforming the WM surface, the pial surface was generated. As a final step, the WM and pial surfaces were averaged together, vertex-by-vertex, to construct a central surface. These central surfaces were then used as basis to determine the degree of cortical gyrification (described below).

\section{CORTICAL GYRIFICATION}

Cortical gyrification was established via calculating mean curvature (Do Carmo, 1976) across thousands of vertices on each individual central surface mesh model. Mean curvature at a given vertex point is defined as:

$$
T_{\text {curvature }}=\sum_{v=1}^{n_{v}}\left(\frac{\left(\bar{x}_{v}-\tilde{x}_{v}\right) \bullet \tilde{N}_{v}}{B_{v}}\right)^{2}
$$

where $\tilde{x}_{v}$ is the centroid of its neighbors of vertex $v, B_{v}$ is the average distance from the centroid of each of the neighbors, and "." is the vector product operator. As demonstrated in Figure 1, the resulting values can be expressed in degrees ranging from $-180^{\circ}$ to $180^{\circ}$, where large negative values correspond to sulci and large positive values to gyri. To increase the signal-to-noise ratio, mean curvature at a given vertex was averaged within a geodesic distance of $3 \mathrm{~mm}$, followed by calculating absolute mean curvature. Finally, a smoothing procedure was applied using a surface-based heat kernel filter (Chung et al., 2005) with a full width at half maximum (FWHM) of $25 \mathrm{~mm}$. The resulting smoothed absolute mean curvature values (hereafter referred to as curvature values) were used as dependent variables in the statistical analyses.

\section{STATISTICAL ANALYSES}

The curvature values were compared at each vertex point between the 50 meditators and 50 controls, while co-varying for gender and age (analysis I). In addition, within the sample of the 50 meditators, correlation analyses were performed to determine associations between curvature values at each vertex point and the number

\footnotetext{
${ }^{2}$ http://surfer.nmr.mgh.harvard.edu
} 


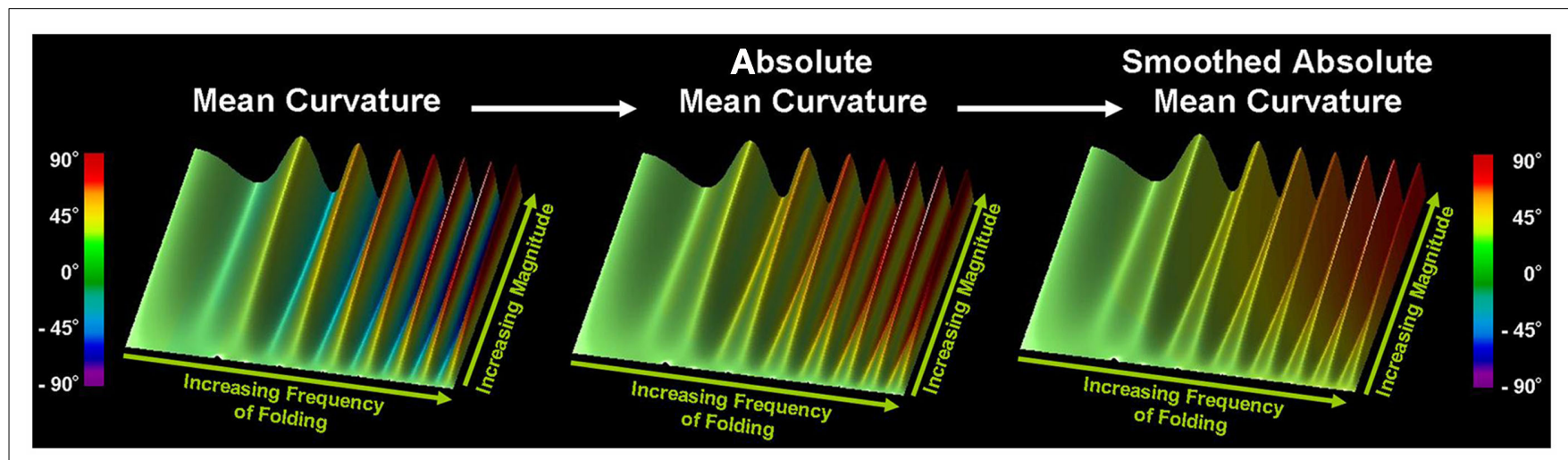

FIGURE 1 | Estimation of cortical gyrification. Illustrated is the computation of local gyrification using a simulated folded surface. The magnitude of the folding increases from proximal to distal, and the frequency increases from left to right. Left Panel: After establishing mean curvature (in degrees), sulci can be identified as regions with large negative values (displayed in blue), while gyri are characterized by large positive values (displayed in red). Middle Panel: After averaging mean curvature within distances of $3 \mathrm{~mm}$, values are transformed into absolute mean curvature (i.e., all values become positive regardless of whether they represent gyri or sulci). Higher values indicate areas with larger gyrification. Right Panel: Curvature values are smoothed using a surface-based heat kernel filter with $\mathrm{FWHM}=25 \mathrm{~mm}$. As demonstrated, increases in the amplitude and wavelength of the simulated folding are reflected in increased values of smoothed absolute mean curvature. of meditation years, while removing the partial effects of age and gender (analysis II). Given our regional hypotheses (and since previous studies with focus on cortical gyrification do not yet exist), significance profiles were established using uncorrected thresholds at $p \leq 0.05$ as well as $p \leq 0.01$. This allows for a first exploratory characterization of such effects and also provides a framework against which outcomes from future analyses can be compared.

\section{RESULTS}

\section{ANALYSIS I: GROUP DIFFERENCES}

As demonstrated in Figure 2 (top panel), cortical gyrification in long-term meditators was larger in numerous regions across the lateral and medial cortices at $p \leq 0.05$ (depicted in yellow/orange). These regions include the left (L) and right (R) anterior dorsal insula, pre/post central gyrus (L), central sulcus (L), inferior/middle temporal gyrus (L), angular gyrus (L), and parieto-occipital fissure (L) as well as parietal operculum (R), fusiform gyrus (R), and cuneus (R). As further illustrated in Figure 2 (bottom panel), when applying stricter significance thresholds $(p \leq 0.01)$, these group differences remained evident within the left precentral gyrus (cluster 1), bilaterally within the anterior dorsal insula (clusters 2-3), the right fusiform gyrus (cluster 4), and the right cuneus (cluster 5). While there were also some clusters indicating larger gyrification in controls at $p \leq 0.05$ (top panel; depicted in cyan), these effects were no longer present (bottom panel) when applying stricter significance criteria $(p \leq 0.01)$. Overall, the global significance maximum was located within the right anterior dorsal insula (red circle) indicating a larger gyrification in meditators compared to controls.

\section{ANALYSIS II: CORRELATIONS}

As demonstrated in Figure 3 (top panel), cortical gyrification appears to increase as the number of meditation years increases. These positive correlations (depicted in yellow/orange) were evident in numerous regions across the left and right lateral surfaces as well as the left medial surface. In contrast, the right medial surface showed predominantly negative correlations (depicted in cyan) suggesting that gyrification decreases as the number of practices years increase. As further illustrated in Figure 3 (bottom panel), when applying stricter significance thresholds, almost all significance clusters indicating significant negative correlations were no longer present. Similarly, only a few clusters indicating significant positive correlations remained evident. However, pronounced positive correlations within the right posterior temporal lobe as well as the right anterior dorsal insula (global maximum) remained at the stricter significance threshold. The spatial location of the maximum correlation effects within the right insula (red circle) corresponds well with the location of the maximum group difference (as shown in Figure 2). No further spatial correspondences between significant correlations (Figure 3) and significant group differences (Figure 2) were observed.

\section{DISCUSSION}

Our study was designed to examine cortical gyrification in a large sample $(n=100)$ of meditators and well-matched controls. When applying more liberal significance thresholds ( $p \leq 0.05)$, gyrification was larger in meditators as well as in controls (depending on the cortical region) in numerous areas across the cortex. Interestingly, meditators showed larger gyrification in some of the regions where prior analyses have revealed thicker cortices (more GM, respectively) in meditators compared to controls. Regions included the right anterior insula (Lazar et al., 2005; Holzel et al., 2008), the left inferior temporal gyrus (Holzel et al., 2008; Luders et al., 2009), the left central sulcus and its vicinity (Luders et al., 2009; Grant et al., 2010), as well as the right parietal operculum, which houses the secondary somato-sensory cortex (Grant et al., 2010). The current study also revealed a larger gyrification of the fusiform gyrus but these effects were located within the right hemisphere, rather than the left hemisphere (Vestergaard-Poulsen et al., 2008). When applying stricter significance thresholds $(p \leq 0.01)$, cortical gyrification was exclusively larger in meditators in five 


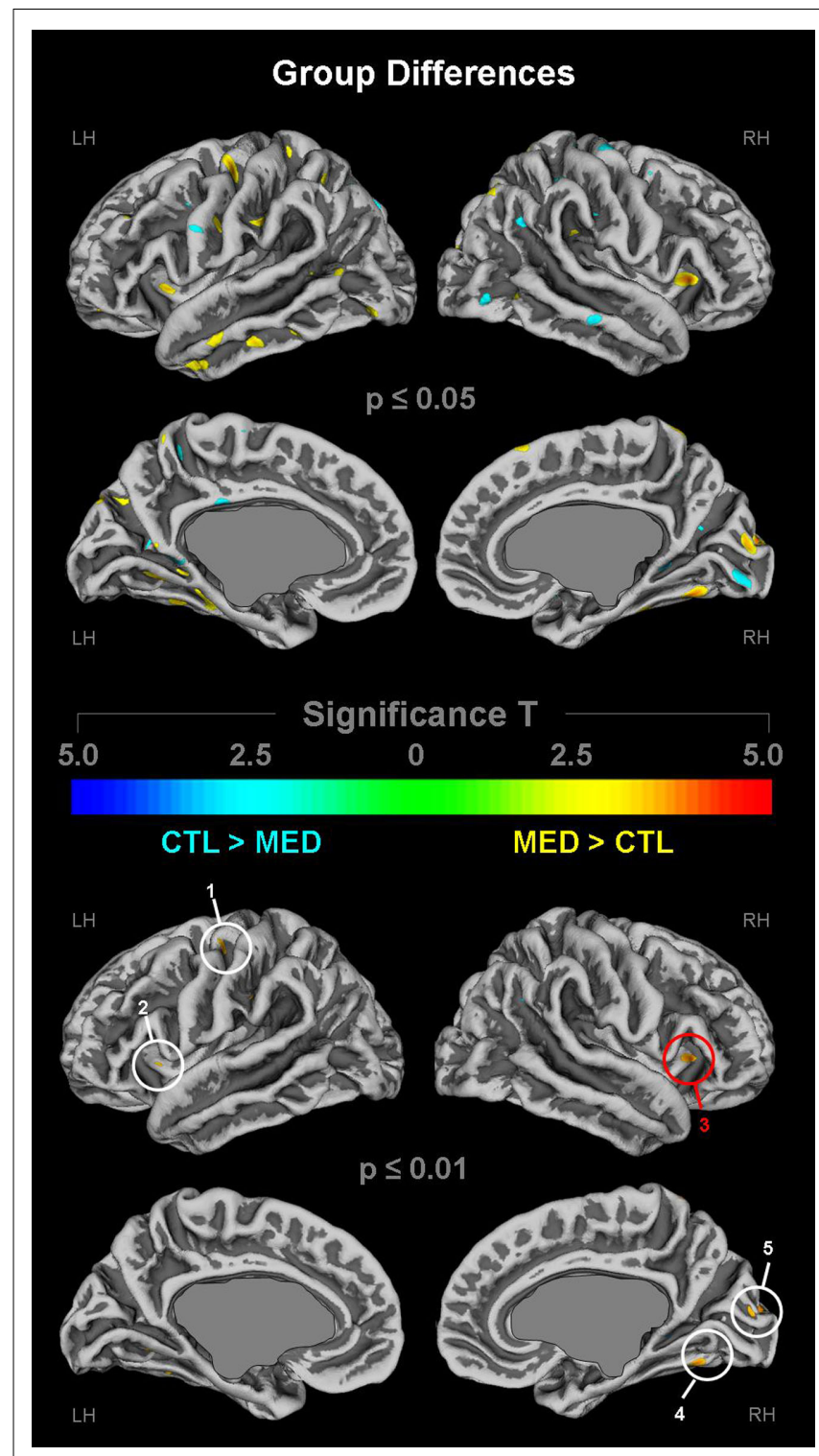

FIGURE 2 | Group differences in cortical gyrification. Shown are group differences at $p \leq 0.05$ (upper panel) and $p \leq 0.01$ (lower panel), uncorrected for multiple comparisons. The color bar encodes significance $(T)$. Areas with larger gyrification in meditators (MED) are depicted in yellow/orange; areas with larger gyrification in controls (CTL) are depicted in cyan. Callosal, subcallosal, and midbrain regions have been excluded on the medial surface maps. Numeric clusters indicate larger gyrification in meditators at $p \leq 0.01$ within (1) left precentral gyrus; (2) left insula; (3) right insula; (4) right fusiform gyrus; (5) right cuneus. The red circle indicates the global maximum. $\mathrm{LH}$, left hemisphere; $\mathrm{RH}$, right hemisphere.

distinct regions, regional observations that were in line with our a priori hypotheses. These regions included the left precentral gyrus, the left and right anterior dorsal insula, the right fusiform gyrus, and the right cuneus. The discussion of the possible functional implications of our findings will thus emphasize these five cortical regions, with particular focus on the right insula (global maximum).

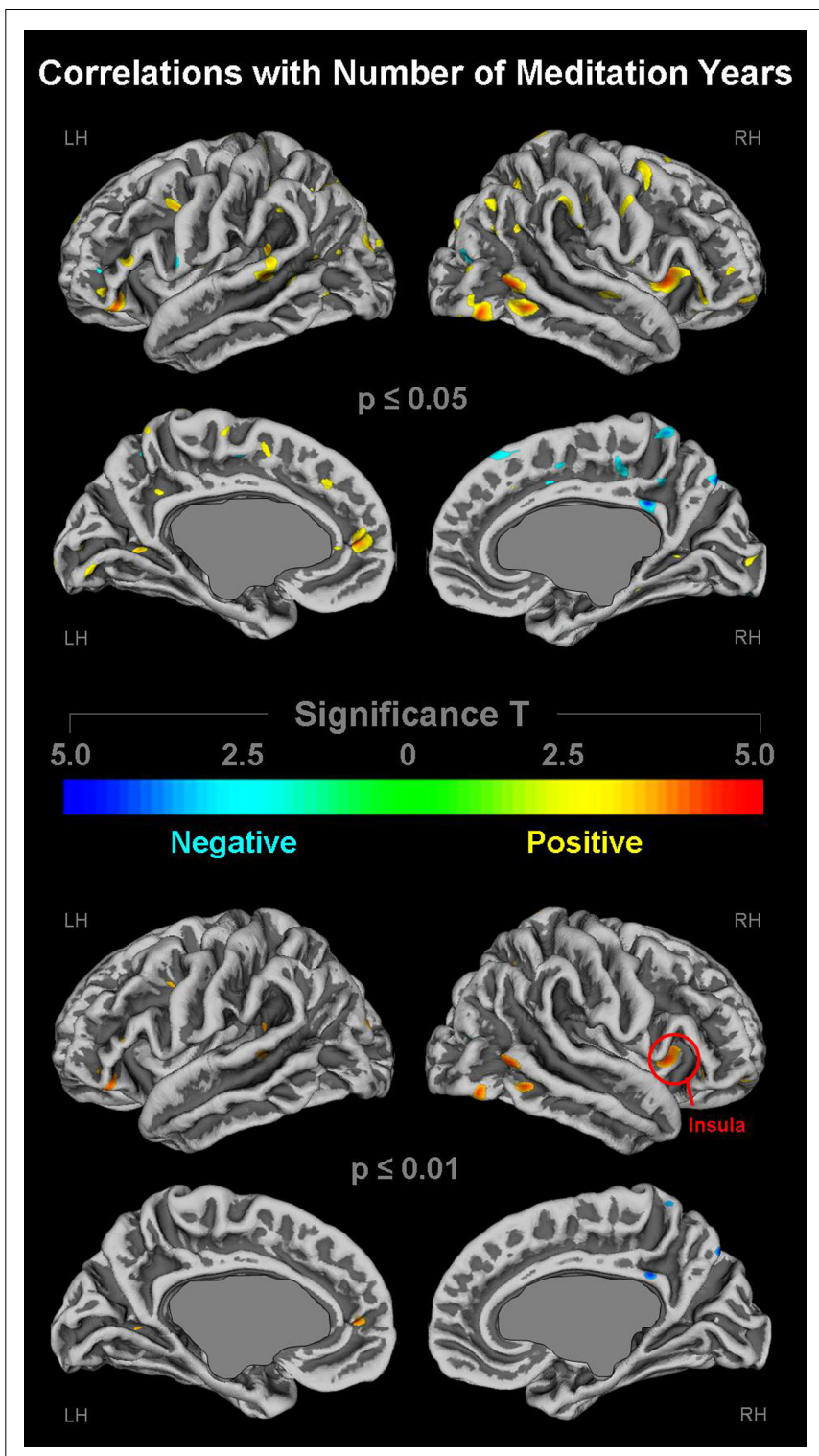

FIGURE 3 | Links between cortical gyrification and number of meditation years. Shown are correlations at $p \leq 0.05$ (upper panel) and $p \leq 0.01$ (lower panel), uncorrected for multiple comparisons. The color bar encodes significance $(T)$. Areas with positive correlations (i.e., more meditation years link with more gyrification) are depicted in yellow/orange; negative correlations (i.e., more meditation years link with less gyrification) are depicted in cyan. Callosal, subcallosal, and midbrain regions have been excluded on the medial surface maps. The red circle indicates the global maximum. $\mathrm{LH}$, left hemisphere; $\mathrm{RH}$, right hemisphere.

\section{INSULAR EFFECTS}

The most pronounced group differences were detected within the right anterior dorsal insula. Moreover, there was a striking spatial correspondence between this region and the outcomes of our correlation analysis that indicated significant positive correlations between insular complexity and meditation practice years. As mentioned above, previous analyses have also exposed structural differences between meditators and non-meditators within 
the right (anterior) insula (Lazar et al., 2005; Holzel et al., 2008). Moreover, functional analyses have revealed an activation of the insula during meditative states and/or onset of meditation (Farb et al., 2007; Lutz et al., 2008; Baerentsen et al., 2010).

Of potential functional relevance to the current findings, the right fronto-insular cortex has been shown to play a critical role in switching between central-executive and default-mode network (DMN; Sridharan et al., 2008). The DMN, a well-characterized resting state network, is most evident in states of daydreaming, mind-wandering, and projections into past or future (Shulman et al., 1997; Raichle et al., 2001; Greicius et al., 2003; Mason et al., 2007; Raichle and Snyder, 2007; Buckner et al., 2008). Since these aforementioned processes are well-known distractions in the context of meditation, successful meditators might be equipped with a better ability to control (i.e., switch on/off) the DMN. Indeed, a recent functional imaging experiment revealed shorter neural responses in regions of the DMN in meditators compared to controls suggesting that "the regular practice of Zen meditation enhances the capacity for voluntary regulation of spontaneous mental activity" (Pagnoni et al., 2008). The altered gyral complexity of the right insula perhaps constitutes one important neural substrate for such ability.

Furthermore, the insula has been recently proposed to integrate a wealth of information, such as interoceptive sensations (within its $\mathrm{mid} /$ posterior parts), which cumulate in a concept of awareness anteriorly (Craig, 2009, 2010). For example, when subjects (unselected for meditation) were asked to judge the timing of their own heart beat relative to feedback notes, the neural activity of the right anterior dorsal insula predicted interoceptive accuracy (Critchley et al., 2004). In addition, interoceptive accuracy as well as subjective ratings of visceral awareness were positively correlated with the local GM of the right anterior dorsal insula. Moreover, a meta-analysis of functional imaging studies provided evidence for overlapping activation bilaterally within the anterior dorsal insula to a wide variety of different stimuli, including cognitive, socio-emotional, olfacto-gustatory, as well as interoception and pain processing (Kurth et al., 2010). Thus, the anterior insula may constitute a "hub" for autonomic, affective, and cognitive integration, as previously suggested (Damasio, 1994; Critchley et al., 2004; Critchley, 2005). Meditators are masters in introspection and awareness as well as in emotional control and self-regulation. Moreover, meditators often practice techniques oriented toward enhancing unconditional, positive emotional states of kindness and compassion (Hofmann et al., 2011). Thus, the altered gyrification of the (right) anterior dorsal insula in meditators might be linked to these singular abilities and/or dedicated practices.

\section{OTHER REGIONS AFFECTED}

Meditators also demonstrated larger gyrification within the left precentral gyrus (Figure 2, cluster 1). Such effects within the primary motor cortex may be more difficult to interpret and further research will be necessary to elucidate the functional relevance of group differences in motor areas of the brain. However, previous analyses (Luders et al., 2009, 2011a) detected similar alterations in meditators, either with respect to the integrity of a fiber tract predominantly originating/terminating in motor areas (corticalspinal tract) or with respect to GM tissue in paracentral regions (the latter when thresholds were lowered to detect significance trends). Moreover, the aforementioned imaging experiment on interoceptive awareness (where subjects had to judge the timing of their heart beat) did not only reveal enhanced insular activity but also enhanced activity of the lateral somatomotor cortex as well as the supplementary motor cortex (Critchley et al., 2004). Thus, regions in the vicinity of the motor cortices (perhaps in close association with the anterior dorsal insula) might aid in supporting interoceptive attention and awareness.

In contrast, structural alterations within the right cuneus (Figure 2, cluster 5) have not been previously reported. However, since the cuneus has been suggested to be heavily involved in shifting or maintaining spatial/visual attention (Corbetta et al., 1998; Hopfinger et al., 2000; Simpson et al., 2011) our findings appear in agreement with prior evidence that meditation is associated with superior visual perception (Brown et al., 1984a,b; Tloczynski et al., 2000; Tha et al., 2007; Kozhevnikov et al., 2009; Hodgins and Adair, 2010). This, in turn, might also be related to the larger gyrification of the right fusiform gyrus (Figure 2, cluster 4), which is part of the ventral visual stream and involved in the processing of visual information, identification, and recognition as well as attention control (Corbetta et al., 1998; Hopfinger et al., 2000; Joseph, 2001; Said et al., 2011). Supporting evidence for the involvement of the fusiform gyrus in the process of meditation has been provided in a previous study revealing larger GM densities in long-term meditators (Vestergaard-Poulsen et al., 2008), although fusiform effects were located within the left hemisphere.

\section{NATURE VERSUS NURTURE}

Given the cross-sectional design of this study, our findings do not allow any definitive causal conclusions. On the one hand, a different cortical gyrification in meditators might constitute an innate brain feature; on the other hand, regular meditation practice might alter gyral/sulcal features of specific brain regions routinely engaged in the activity of meditating. In general, it is estimated that about $30 \%$ of phenotypic variance in gyrification is attributed to genetic variation (Rogers et al., 2010). Although the folding pattern of the cortical surface is assumed to be largely under the influence of genetic control (Armstrong et al., 1995; Piao et al., 2004), the degree of folding has been demonstrated to still change in childhood and adolescence (Blanton et al., 2001). This suggests that environmental factors may have a modulating impact on cortical gyrification (Bartley et al., 1997; Lohmann et al., 1999; White et al., 2010). That is, actively meditating (especially regularly meditating over a long period of time) may induce plastic changes on a microanatomical level (synaptic pruning, dendritic arborization, etc.), which conceivably could alter the morphology of gyri and sulci and impact the folding of the cortical surface (Van Essen, 1997). The observed positive correlations between gyrification and number of practice years add some further support to suggest that meditation enhances regional gyrification.

It is equally plausible that the observed effects exist due to an interaction between nature and nurture. That is, people with a specific pattern of normal anatomical variability might have picked up meditation and induced further structural changes in the course of their long-term practice. It is similarly likely, that different mechanisms apply to different brain regions. For example, training and practice might have a stronger impact on cerebral regions that mature later. Thus, meditation-induced alterations may especially 
occur in superficial or tertiary sulci, which develop mainly after birth and appear to be more affected by non-genetic influences (Lohmann et al., 1999). In contrast, deeper fissures and developmentally earlier forming sulci are more strongly influenced by genetic processes and thus might be much less susceptible to environmental perturbations. Interestingly, insular sulci (as well as the central sulci) are among the first macroscopical structures identified on the lateral surface of the human fetal brain (with earlier development in the right hemisphere than in the left; Afif et al., 2007). Since the folding pattern of the insula appears to be established around birth, it is likely that the observed group differences within the insula (as well as in the vicinity of the central sulcus) constitute innate cortical characteristics. In agreement with this assumption, cross-sectional studies detected insular differences in meditators (Lazar et al., 2005; Holzel et al., 2008), while longitudinal analyses with particular focus on the insula did not reveal any meditation-induced insular changes (Holzel et al., 2011). Nevertheless, although Holzel's longitudinal study (2011) did not detect any changes of insular GM within the time frame of 8 weeks, it is likely that such changes occur with respect to other insular characteristics and/or as a consequence of longer lasting practices (note, meditators in the current study had practiced almost 20 years on average). Moreover, the longitudinal study (Holzel et al., 2011) included participants of a mindfulness-based stress reduction program and hence a selective sample with a particular health condition. Therefore, the relevance of these findings to ours is less direct and it may as well be possible that insular alterations in long-term meditation practitioners constitute practice-induced effects. In support of this hypothesis, insular complexity seems to increase as the number of meditation years increases, as revealed in our correlation analysis. Similar findings were reported from another cross-sectional study (Holzel et al., 2008) demonstrating a trend toward significant positive correlations between insular GM and the amount of meditation practice (i.e., hours of meditation training). Notwithstanding, further research on links between meditation practices and cortical gyrification is necessary, and any conclusions with respect to the possible determinants of the observed group differences and correlations remain speculative.

\section{SUMMARY AND OUTLOOK}

For the first time, the current study examined cortical gyrification in association with mindfulness practices. The group differences between meditators and controls as well as the links between local gyrification and number of meditation years point to cortical regions involved in processes related to meditation, with particular involvement of the (right) anterior dorsal insula. Since cortical

\section{REFERENCES}

Afif, A., Bouvier, R., Buenerd, A., Trouillas, J., and Mertens, P. (2007). Development of the human fetal insular cortex: study of the gyration from 13 to 28 gestational weeks. Brain Struct. Funct. 212, 335-346.

Armstrong, E., Schleicher, A., Omran, H., Curtis, M., and Zilles, K. (1995). The ontogeny of human gyrification. Cereb. Cortex 5, 56-63.

Awate, S. P., Yushkevich, P. A., Song, Z., Licht, D. J., and Gee, J. C. (2010).

gyrification relates to behavioral traits in humans (Awate et al., 2010), the observed alterations in long-term practitioners might reflect specific traits associated with meditation (e.g., higher levels of introspection, awareness, response control, compassion, etc.). However, this hypothesis remains to be tested with actual behavioral data and/or performance measures in follow-up studies. Moreover, the applied curvature measurement does not allow distinguishing between sulci and gyri (nor between a cortical region with a large number of shallow convolutions and one with only a small number of deep convolutions). Thus, future studies may consider introducing additional curvature indices to improve the regional specificity in general and also to discriminate between sulcal and gyral effects. Increasing the spatial resolution of findings will provide important clues, for example, with respect to whether primary, secondary, or tertiary sulci are affected (which may provide hints with respect to the heredity of effects). Similarly, being able to discriminate between gyral and sulcal effects may provide hints with respect to a possible neuroprotective impact in the context of brain aging, as previously discussed (Lazar et al., 2005; Pagnoni and Cekic, 2007; Luders et al., 2011a). For example, it was shown that the shape of gyri/sulci changes due to normal aging processes, with gyri getting more sharply and steeply curved and sulci becoming flatter (Magnotta et al., 1999). If meditation had a brain-preserving effect, meditators might have wider gyral crowns and/or narrower sulci than age-matched controls. Last but not least, since there seems to be a positive correlation between cortical gyrification and intelligence (Luders et al., 2008), controlling for IQ in future studies, will ensure that individual intelligence is not a significant modulator when comparing meditators and controls with respect to gyrification.

\section{ACKNOWLEDGMENTS}

We warmly thank all meditators for their participation in our study. For generous support the authors thank the Brain Mapping Medical Research Organization, the Robson Family and Northstar Fund, and the following Foundations: Brain Mapping Support, Pierson-Lovelace, Ahmanson, Tamkin, William M., and Linda R. Dietel Philanthropic Fund at the Northern Piedmont Community, Jennifer Jones-Simon, and Capital Group Companies. This study was additionally supported by the NIH, including NIMH (MH092301) and NCRR (RR013642, RR12169, and RR00865), as well as NIBIB, NIDA, NINDS, and NCI. Further support was provided by grants from the Human Brain Project (P20-MHDA52176 and 5P01-EB001955). Last but not least, the authors are grateful to Trent Thixton who assisted with the acquisition of the image data.

variability of human brain size and cortical gyral patterns. Brain $120(\mathrm{Pt}$ 2), 257-269.

Blanton, R. E., Levitt, J. G., Thompson, P. M., Narr, K. L., CapetilloCunliffe, L., Nobel, A., Singerman, J. D., McCracken, J. T., and Toga, A. W. (2001). Mapping cortical asymmetry and complexity patterns in normal children. Psychiatry Res. 107 29-43.

Brown, D., Forte, M., and Dysart, M. (1984a). Differences in visual sensitivity among mindfulness meditators and non-meditators. Percept. Mot. Skills 58, 727-733.

Brown, D., Forte, M., and Dysart, M. (1984b). Visual sensitivity and mindfulness meditation. Percept. Mot. Skills 58, 775-784.

Buckner, R. L., Andrews-Hanna, J. R., and Schacter, D. L. (2008). The brain's default network: anatomy, function, and relevance to disease. Ann. N. Y. Acad. Sci. 1124, $1-38$. 
Chung, M. K., Robbins, S. M., Dalton, K. M., Davidson, R. J., Alexander, A. L., and Evans, A. C. (2005). Cortical thickness analysis in autism with heat kernel smoothing. Neuroimage 25, 1256-1265.

Corbetta, M., Akbudak, E., Conturo, T. E., Snyder, A. Z., Ollinger, J. M., Drury, H. A., Linenweber, M. R., Petersen, S. E., Raichle, M. E., Van, E., and Shulman, G. L. (1998). A common network of functional areas for attention and eye movements. Neuron 21, 761-773.

Craig, A. D. (2009). How do you feelnow? The anterior insula and human awareness. Nat. Rev. Neurosci. 10, 59-70.

Craig, A. D. (2010). The sentient self. Brain Struct. Funct. 214, 563-577.

Critchley, H. D. (2005). Neural mechanisms of autonomic, affective, and cognitive integration. J. Comp. Neurol. 493, 154-166.

Critchley, H. D., Wiens, S., Rotshtein, P., Ohman, A., and Dolan, R. J. (2004). Neural systems supporting interoceptive awareness. Nat. Neurosci. 7, 189-195.

Dale, A. M., Fischl, B., and Sereno, M. I. (1999). Cortical surface-based analysis. I. Segmentation and surface reconstruction. Neuroimage 9, 179-194.

Damasio, A. R. (1994). Descartes' error and the future of human life. Sci. Am. 271, 144.

Do Carmo, M. P. (1976). Differential Geometry of Curves and Surfaces. Prentice Hall, NJ: Englewood Cliffs.

Farb, N. A., Segal, Z. V., Mayberg, H., Bean, J., McKeon, D., Fatima, Z., and Anderson, A. K. (2007). Attending to the present: mindfulness meditation reveals distinct neural modes of selfreference. Soc. Cogn. Affect. Neurosci. 2, 313-322.

Fischl, B., Sereno, M. I., and Dale, A. M. (1999a). Cortical surface-based analysis. II: Inflation, flattening, and a surface-based coordinate system. Neuroimage 9, 195-207.

Fischl, B., Sereno, M. I., Tootell, R. B., and Dale, A. M. (1999b). High-resolution intersubject averaging and a coordinate system for the cortical surface. Hum. Brain Mapp. 8, 272-284.

Gaser, C., Luders, E., Thompson, P. M., Lee, A. D., Dutton, R. A., Geaga, J. A., Hayashi, K. M., Bellugi, U., Galaburda, A. M., Korenberg, J. R., Mills, D. L., Toga, A. W., and Reiss, A. L. (2006). Increased local gyrification mapped in Williams syndrome. Neuroimage 33, 46-54.
Grant, J. A., Courtemanche, J., Duerden, E. G., Duncan, G. H., and Rainville, P. (2010). Cortical thickness and pain sensitivity in Zen meditators. Emotion 10, 43-53.

Greicius, M. D., Krasnow, B., Reiss, A. L., and Menon, V. (2003). Functional connectivity in the resting brain: a network analysis of the default mode hypothesis. Proc. Natl. Acad. Sci. U.S.A. 100, 253-258.

Hodgins, H. S., and Adair, K. C. (2010). Attentional processes and meditation. Conscious. Cogn. 19, 872-878.

Hofmann, S. G., Grossman, P., and Hinton, D. E. (2011). Lovingkindness and compassion meditation: potential for psychological interventions. Clin. Psychol. Rev. 31, 1126-1132.

Holzel, B. K., Carmody, J., Evans, K. C., Hoge, E. A., Dusek, J. A., Morgan, L., Pitman, R. K., and Lazar, S. W. (2010). Stress reduction correlates with structural changes in the amygdala. Soc. Cogn. Affect. Neurosci. 5, 11-17.

Holzel, B. K., Carmody, J., Vangel, M., Congleton, C., Yerramsetti, S. M., Gard, T., and Lazar, S. W. (2011). Mindfulness practice leads to increases in regional brain gray matter density. Psychiatry Res. 191, 36-43.

Holzel, B. K., Ott, U., Gard, T., Hempel, H., Weygandt, M., Morgen, K., and Vaitl, D. (2008). Investigation of mindfulness meditation practitioners with voxel-based morphometry. Soc. Cogn. Affect. Neurosci. 3, 55-61.

Hopfinger, J. B., Buonocore, M. H., and Mangun, G. R. (2000). The neural mechanisms of top-down attentional control. Nat. Neurosci. 3, 284-291.

Jha, A. P., Krompinger, J., and Baime, M. J. (2007). Mindfulness training modifies subsystems of attention. Cogn. Affect. Behav. Neurosci. 7, 109-119.

Joseph, J. E. (2001). Functional neuroimaging studies of category specificity in object recognition: a critical review and meta-analysis. Cogn. Affect. Behav. Neurosci. 1, 119-136.

Kozhevnikov, M., Louchakova, O., Josipovic, Z., and Motes, M. A. (2009). The enhancement of visuospatial processing efficiency through Buddhist Deity meditation. Psychol. Sci. 20, 645-653.

Kurth, F., Zilles, K., Fox, P. T., Laird, A. R., and Eickhoff, S. B. (2010). A link between the systems: functional differentiation and integration within the human insula revealed by metaanalysis. Brain Struct. Funct. 214 519-534.

Lazar, S. W., Kerr, C. E., Wasserman, R. H., Gray, J. R., Greve, D. N., Treadway, M. T., McGarvey, M., Quinn, B. T., Dusek, J. A., Benson, H. Rauch, S. L., Moore, C. I., and Fischl, B. (2005). Meditation experience is associated with increased cortical thickness. Neuroreport 16 1893-1897.

Lohmann, G., von Cramon, D. Y., and Steinmetz, H. (1999). Sulcal variability of twins. Cereb. Cortex 9, 754-763.

Luders, E., Clark, K., Narr, K. L., and Toga, A. W. (2011a). Enhanced brain connectivity in long-term meditation practitioners. Neuroimage 57, 1308-1316.

Luders, E., Phillips, O., Clark, K., Kurth, F., Toga, A., and Narr, K. (2011b). Bridging the hemispheres in meditation: thicker callosal regions and enhanced fractional anisotropy (FA) in long-term practitioners. $\mathrm{Neu}$ roimage.

Luders, E., Narr, K. L., Bilder, R. M., Szeszko, P. R., Gurbani, M N., Hamilton, L., Toga, A. W. and Gaser, C. (2008). Mapping the relationship between cortical convolution and intelligence: effects of gender. Cereb. Cortex 18, 2019-2026.

Luders, E., Thompson, P. M., Narr, K. L., Toga, A. W., Jancke, L., and Gaser, C. (2006). A curvature-based approach to estimate local gyrification on the cortical surface. Neuroimage 29, 1224-1230.

Luders, E., Toga, A. W., Lepore, N., and Gaser, C. (2009). The underlying anatomical correlates of long-term meditation: larger hippocampal and frontal volumes of gray matter. Neuroimage 45 672-678.

Lutz, A., Brefczynski-Lewis, J., Johnstone, T., and Davidson, R. J. (2008). Regulation of the neural circuitry of emotion by compassion meditation: effects of meditative expertise. PLoS ONE 3, e1897. doi:10.1371/journal.pone.0001897

Magnotta, V. A., Andreasen, N. C. Schultz, S. K., Harris, G., Cizadlo, T., Heckel, D., Nopoulos, P., and Flaum, M. (1999). Quantitative in vivo measurement of gyrification in the human brain: changes associated with aging. Cereb. Cortex 9, 151-160.

Mason, M. F., Norton, M. I., Van Horn, J. D., Wegner, D. M., Grafton, S. T., and Macrae, C. N. (2007). Wandering minds: the default network and stimulus-independent thought. Science 315, 393-395.

Pagnoni, G., and Cekic, M. (2007). Age effects on gray matter volume and attentional performance in Zen meditation. Neurobiol. Aging 28, 1623-1627.

Pagnoni, G., Cekic, M., and Guo, Y. (2008). "Thinking about notthinking": neural correlates of conceptual processing during Zen meditation. PLoS ONE 3:e3083. doi:10.1371/journal.pone.0003083

Piao, X., Hill, R. S., Bodell, A., Chang, B. S., Basel-Vanagaite, L., Straussberg, R., Dobyns, W. B., Qasrawi, B., Winter, R. M., Innes, A. M., Voit, T., Ross, M. E., Michaud, J. L., Descarie, J. C., Barkovich, A. J., and Walsh, C. A. (2004). G proteincoupled receptor-dependent development of human frontal cortex. Science 303, 2033-2036.

Raichle, M. E., MacLeod, A. M., Snyder, A. Z., Powers, W. J., Gusnard, D. A., and Shulman, G. L. (2001). A default mode of brain function. Proc. Natl. Acad. Sci. U.S.A. 98, 676-682.

Raichle, M. E., and Snyder, A. Z. (2007). A default mode of brain function: a brief history of an evolving idea. Neuroimage 37, 1083-1090.

Rogers, J., Kochunov, P., Zilles, K., Shelledy, W., Lancaster, J., Thompson, P., Duggirala, R., Blangero, J., Fox, P. T., and Glahn, D. C. (2010). On the genetic architecture of cortical folding and brain volume in primates. Neuroimage 53, 1103-1108.

Said, C. P., Haxby, J. V., and Todorov, A. (2011). Brain systems for assessing the affective value of faces. Philos. Trans. R. Soc. Lond. B Biol. Sci. 366, 1660-1670.

Shulman, G. L., Fiez, J. A., Corbetta, M., Buckner, R. L., Miezin, F. M., Raichle, M. E., and Petersen, S. E. (1997). Common blood flow changes across visual Tasks: II. Decreases in cerebral cortex. J. Cogn. Neurosci. 9, 648-663.

Simpson, G. V., Weber, D. L., Dale, C. L., Pantazis, D., Bressler, S. L., Leahy, R. M., and Luks, T. L. (2011). Dynamic activation of frontal, parietal, and sensory regions underlying anticipatory visual spatial attention. J. Neurosci. 31, 13880-13889.

Sridharan, D., Levitin, D. J., and Menon, V. (2008). A critical role for the right fronto-insular cortex in switching between central-executive and default-mode networks. Proc. Natl. Acad. Sci. U.S.A. 105, 12569-12574. 
Tang, Y. Y., Lu, Q., Geng, X., Stein, E. A., Yang, Y., and Posner, M. I. (2010). Short-term meditation induces white matter changes in the anterior cingulate. Proc. Natl. Acad. Sci. U.S.A. 107, 15649-15652.

Tloczynski, J., Santucci, A., and AstorStetson, E. (2000). Perception of visual illusions by novice and longerterm meditators. Percept. Mot. Skills 91, 1021-1026.

Van Essen, D. C. (1997). A tension-based theory of morphogenesis and compact wiring in the central nervous system. Nature 385, 313-318.
Vestergaard-Poulsen, P., van, B. M., Skewes, J., Bjarkam, C. R. Stubberup, M., Bertelsen, J., and Roepstorff, A. (2008). Long-term meditation is associated with increased gray matter density in the brain stem. Neuroreport 20, 170-174.

White, T., Su, S., Schmidt, M., Kao, C. Y., and Sapiro, G. (2010). The development of gyrification in childhood and adolescence. Brain Cogn. 72, 36-45.

Zilles, K., Armstrong, E., Schleicher, A., and Kretschmann, H. J. (1988). The human pattern of gyrification in the cerebral cortex. Anat. Embryol. 179, 173-179.

Conflict of Interest Statement: The authors declare that the research was conducted in the absence of any commercial or financial relationships that could be construed as a potential conflict of interest.

Received: 09 November 2011; accepted: 14 February 2012; published online: 29 February 2012.
Citation: Luders E, Kurth F, Mayer EA, Toga AW, Narr KL and Gaser C (2012) The unique brain anatomy of meditation practitioners: alterations in cortical gyrification. Front. Hum. Neurosci. 6:34. doi: 10.3389/fnhum.2012.00034

Copyright $\odot 2012$ Luders, Kurth, Mayer, Toga, Narr and Gaser. This is an openaccess article distributed under the terms of the Creative Commons Attribution Non Commercial License, which permits non-commercial use, distribution, and reproduction in other forums, provided the original authors and source are credited. 


\section{APPENDIX}

Table A1 | Subject-specific meditation practices

\begin{tabular}{|c|c|c|c|c|}
\hline$M$ & Experience (in years) & Frequency (times per week) & Duration (minutes per session) & Meditation style (self-reported) \\
\hline 2 & 5 & 7 & 10 & Samatha \\
\hline 4 & 7 & 3 & 40 & Zen \\
\hline 5 & 7 & 7 & 60 & Kriya \\
\hline 6 & 7 & $3-4$ & 60 & Vipassana \\
\hline 8 & 7 & 7 & 120 & Vipassana \\
\hline 9 & 9 & 7 & 60 & Tibetan Buddhist Meditation \\
\hline 10 & 9 & 3 & 40 & Vipassana \\
\hline 11 & 10 & 7 & 60 & Samatha, Vipassana, Zen \\
\hline 12 & 10 & 3 & 20 & Kriya \\
\hline 13 & 10 & 7 & 60 & Vipassana \\
\hline 17 & 13 & $5-6$ & 60 & Zen \\
\hline 18 & 14 & $1-2$ & 30 & Raja Yoga Meditation \\
\hline 19 & 15 & 7 & 30 & Zen \\
\hline 20 & 15 & 7 & 30 & Samatha, Vipassana \\
\hline 21 & 15 & 7 & 15 & Not specified \\
\hline 22 & 15 & 7 & 45 & Vipassana \\
\hline 23 & 16 & $3-5$ & 90 & Zen \\
\hline 24 & 16 & 7 & 30 & Not specified \\
\hline 25 & 16 & $5-6$ & 30 & Vipassana \\
\hline 26 & 16 & 7 & 60 & Vipassana, Zen \\
\hline 27 & 17 & 7 & 30 & Vajrayana \\
\hline 35 & 23 & 7 & 35 & Vipassana \\
\hline 36 & 25 & 3 & 30 & Vipassana \\
\hline 37 & 28 & 7 & 120 & Dzogchen, Vipassana \\
\hline 38 & 30 & 3 & 30 & Zen \\
\hline 39 & 31 & 7 & 60 & Sadhana, Samatha, Vipassana, \\
\hline 40 & 31 & 7 & 60 & Vipassana, Zen \\
\hline 41 & 32 & 7 & 60 & Dzogchen \\
\hline 42 & 33 & 7 & 60 & Vipassana \\
\hline 43 & 36 & 6 & 20 & Zen \\
\hline 44 & 36 & 7 & 45 & Dzogchen, Mahamudra, Vipassana \\
\hline 45 & 38 & 1 & 60 & Not specified \\
\hline 46 & 38 & 3 & 90 & Chenrezig \\
\hline 47 & 38 & 7 & 150 & Dzogchen, Vajrayana \\
\hline 48 & 41 & 3 & 60 & Samatha, Vipassana \\
\hline 49 & 41 & 1 & 60 & Not specified \\
\hline 50 & 46 & 7 & 45 & Kundalini \\
\hline
\end{tabular}

M: meditators 1-50. 